\title{
Migración intrapélvica (cut-in) en un clavo Gamma. Caso Clínico
}

\author{
A. CAÑELLAS TROBAT ${ }^{1}$, L. SOUCHEIRON ${ }^{1}$, F. ALCAIDE$^{2}$, F.J. FERNÁNDEZ ${ }^{3}$, A. CAÑELLAS RUESGA ${ }^{4}$. \\ ${ }^{1}$ Servicio de Cirugía Ortopédica y Traumatología. \\ ${ }^{2}$ Servicio de Cirugía General. \\ ${ }^{3}$ Catedrático de Universidad. Alcalá de Henares. Madrid. \\ ${ }^{4}$ Servicio de Rehabilitación.
}

\begin{abstract}
Resumen
Una de las complicaciones de los clavos intramedulares es sin duda la implantación central del tornillo cérvico-cefálico. Aún así, la confluencia de diversos factores (osteoporosis, trazo de la fractura, reducción insuficiente del foco, motorización reiterada, etc.) pueden precipitar la salida de éste (cut-out) que viene a evidenciar una prevalencia del 2 al 4\% de las diversas series y, ulterior aflojamiento del montaje. La presencia del cut-in, supone una eventualidad muy ocasional por debajo del 0,3\% de la literatura. En este caso se ilustra una consolidación definitiva afortunada, tras un abanico de factores mecánicos y estructurales que encaminaron a una doble cirugía terapéutica. Se analizan los factores biomecánicos implicados en la génesis del cut-in.
\end{abstract}

Palabras clave: Clavo intramedular, cut-in, fractura cadera, osteoporosis, aflojamiento osteosintesis, laparotomía.

\begin{abstract}
One common complication of intramedullary nails is the central implantation of the cervicocephalic screw. However, the confluence of some factors (osteoporosis, fracture line, inadequate focus reduction, repeated motorizing, etc.) can hasten a cut-out, showing a prevalence of 2 to $4 \%$ of the diverse series, and further assembly loosening. The presence of cut-in is an infrequent eventuality under the $0.3 \%$ of literature. The present case illustrates a fortunate definite consolidation, after a range of mechanical an structural factors leading to a double therapeutic surgery. The biomechanical factors affecting the cut-in origin are analysed.
\end{abstract}

Keywords: Intramedullary nail, cut-in, hip fracture, osteoporosis, osteosynthesis loosening, laparotomy.

\section{Introducción}

Las fracturas de cadera osteoporóticas suponen unas cifras alrededor de unas 60.000 al año en España, según el estudio A.F.O.E. (Acta de fracturas osteoporóticas españolas) [14] realizado por la SECOT (Soc. Española de Cirugía Ortopédica y Traumatología). De ellas, el $91 \%$ corresponden a personas de más de 65 años; a partir de los 85 años

Correspondencia:

Antonio Cañellas

Hospital General Mateu Orfila.

Av., Ronda de Malbuger s/n

Código Postal 07701 - Mahón. Menorca (Illes Balears)

E-mail: zonox@telefonica.net

Teléfono: 971.367 .823 - 971.487 .000 esta cifra se duplica y hemos pasado de una edad media de 73 años a más de 80 años en la última década.

La mortalidad intrahospitalaria por fractura de cadera en nuestro país se sitúa entre el 4 y el $8 \%$, alcanzando al 30\% de los pacientes en los 12 meses siguientes a la fractura, a partir de los 80 años.

Después de sufrir una fractura de cadera, aproximadamente el $50 \%$ no puede caminar y más del $50 \%$ son incapaces de vivir independientemente.

Las complicaciones son frecuentes y diversas $[3,5,13,14]$ fundamentalmente médicas durante el ingreso hospitalario; sin embargo, de nuestra casuística constatamos este único caso de cut-in que está migrado medialmente, no exento de morbilidad aunque con un aceptable resultado final. 
La trascendencia de estas fracturas queda patente en la frase de Cleveland quien hace más de cuarenta años decía que "venimos al mundo a través de la pelvis y lo dejamos por el cuello de fémur".

\section{Caso Clínico}

Ingresa por Urgencias una mujer de 76 años tras caída casual en su domicilio, presentando una fractura conminuta pertrocantérea de cadera derecha tipo 31.A2.1 de la clasificación AO (figura 1) y se aplica tracción blanda al ingreso.

Se interviene de urgencia, realizándose reducción en mesa de tracción y la implantación de un clavo tipo Gamma $3130^{\circ}$, intramedular 11/180 corto al uso. En el control peroperatorio y postoperatorio se evaluó un montaje estable y satisfactorio (figura 2). La paciente fue dada de alta hospitalaria sin complicaciones e inició el protocolo habitual domiciliario, con carga parcial a los 10 días en andador durante las sucesivas semanas, etc. Sin incidencias en el proceso de fisioterapia funcional asistida, si bien su evolución fue lenta y con dolor tolerable, informándonos el fisioterapeuta a las 5 semanas de una limitación parcial coxofemoral pasiva, un acortamiento de la extremidad compensado y crujidos rotacionales.

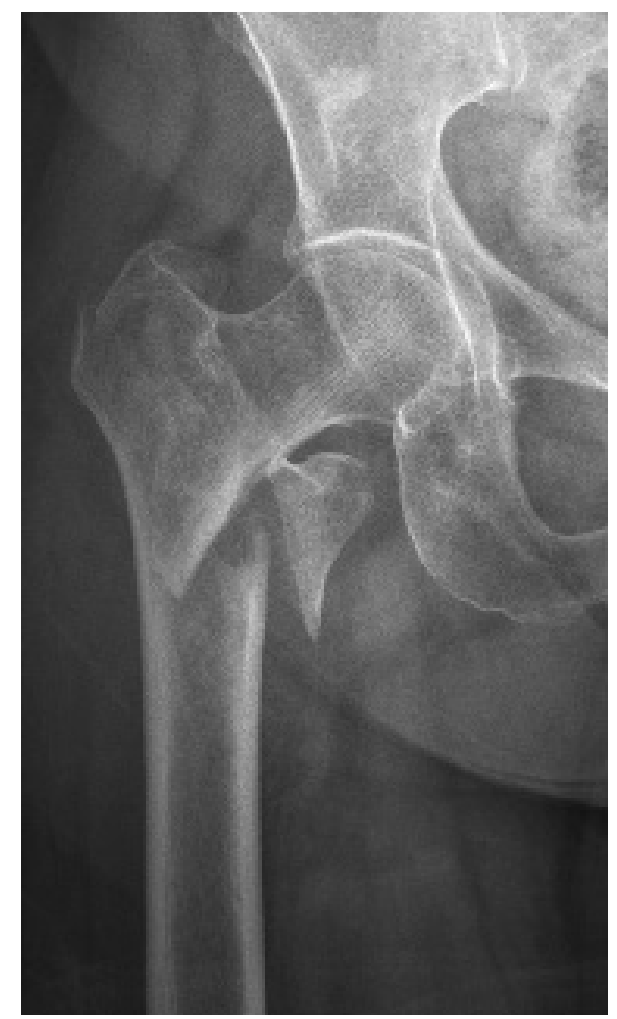

Acude a nuestra consulta externa al mes y medio y practicamos unas radiografías de control con la evidencia de un desbloqueo en el montaje del dispositivo y, migración del tornillo cefálico en dirección centrípeta que invade el coxal -se marca una tenue imagen osteogénica en la línea arqueada y eminencia iliopúbica del mismo- (figura 3). Se presenta con una consolidación bastante estructurada, subtotal, con varización secundaria grave y acortamiento relevante de la extremidad.

En la intervención quirúrgica de rescate bajo fluoroscopia peroperatoria, se extrae el clavo IM con los tornillos menores; se presenta un deslizamiento espontáneo y escurridizo del tornillo cefálico hacia la cavidad intrapélvica al mínimo intento de agarre o pellizco de su borde metálico, hasta el extremo de quedar suspendido en el neo-foramen del coxal (figura 4). Ante tal eventualidad, desistimos de "perseguir" en este acto quirúrgico su rescate.

Se comprueba la consolidación viciosa del foco fractuario original, con una integridad cefálica increíble que, aunque osteoporótica no presenta signos de algodistrofia ósea, hundimiento esférico, ni de necrosis capital.

Ante la necesidad de practicar laparotomía abdominal de rescate a las pocas horas, se complementó el estudio con una TAC pélvica, que es-

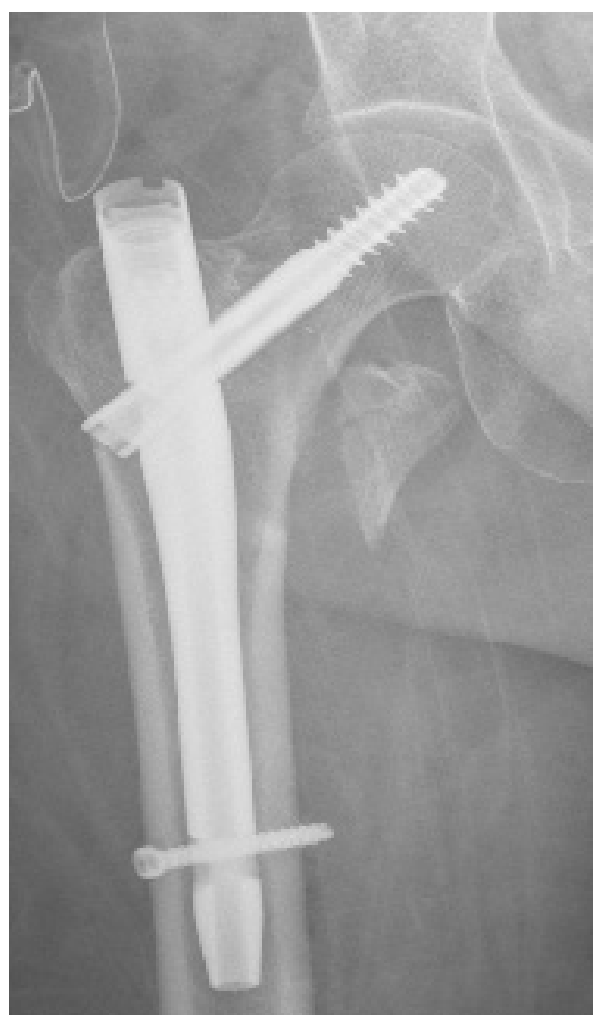

Figura 1 (izquierda). Fractura pertrocantérea al ingreso.

Figura 2 (derecha). Imagen postoperatoria del montaje. 
tableció la trayectoria de aquél en relación a las estructuras blandas abdominales y la localización de la ventana ósea. La conclusión de la TAC helicoidal fue según el informe: "clavo intrapélvico que desplaza el sigma y las estructuras adyacen- tes, existiendo neumoperitoneo rodeando el extremo distal de dicho clavo..."

En las proyecciones 3D (figuras 5 a y 5 b) queda más que patente la trayectoria y la ventana ósea que formó en el área antedicha con la entrada casi
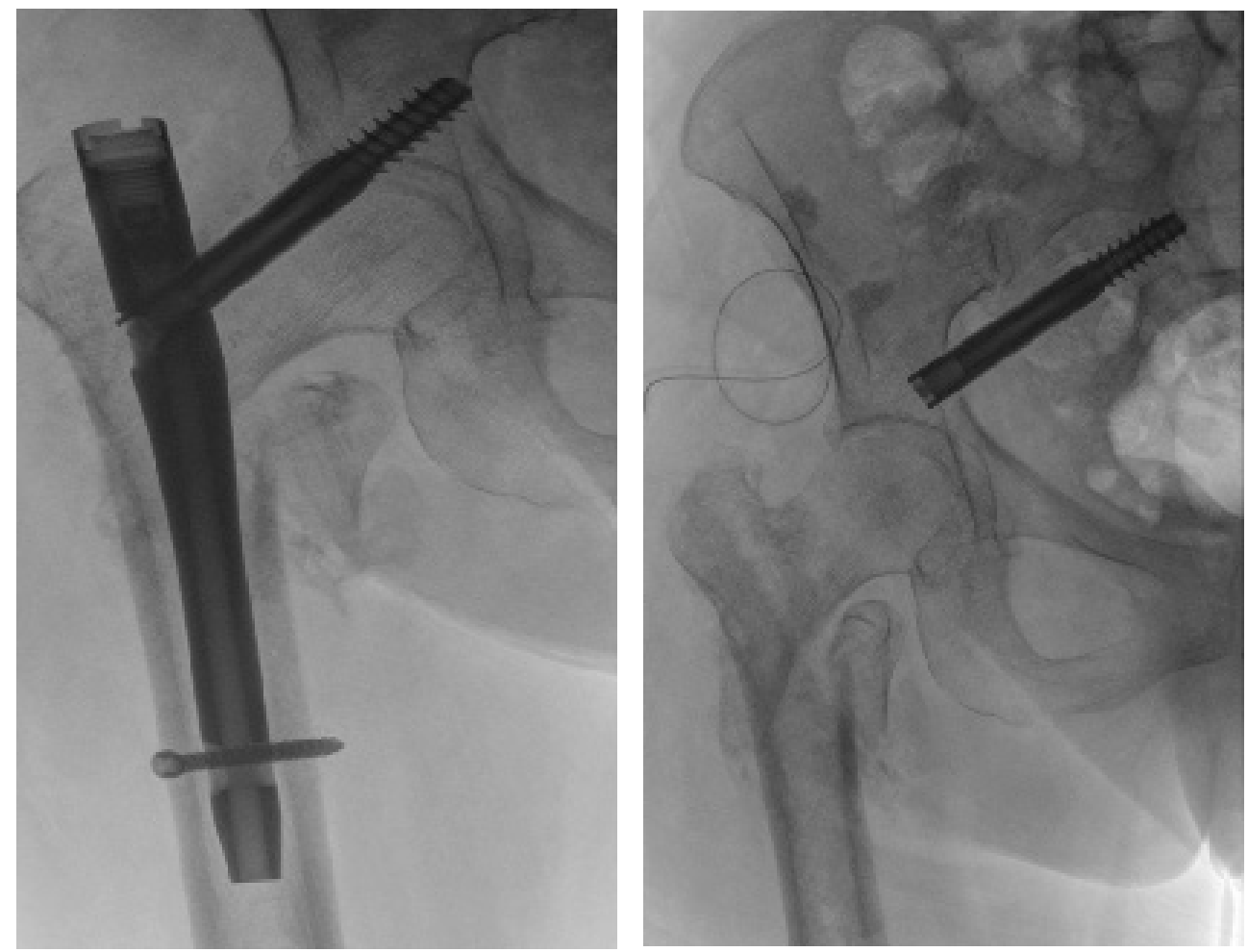

Figura 3 (izquierda). Control a las cinco semanas de la cirugía.

Figura 4 (derecha). Imagen tras el rescate del clavo endomedular.

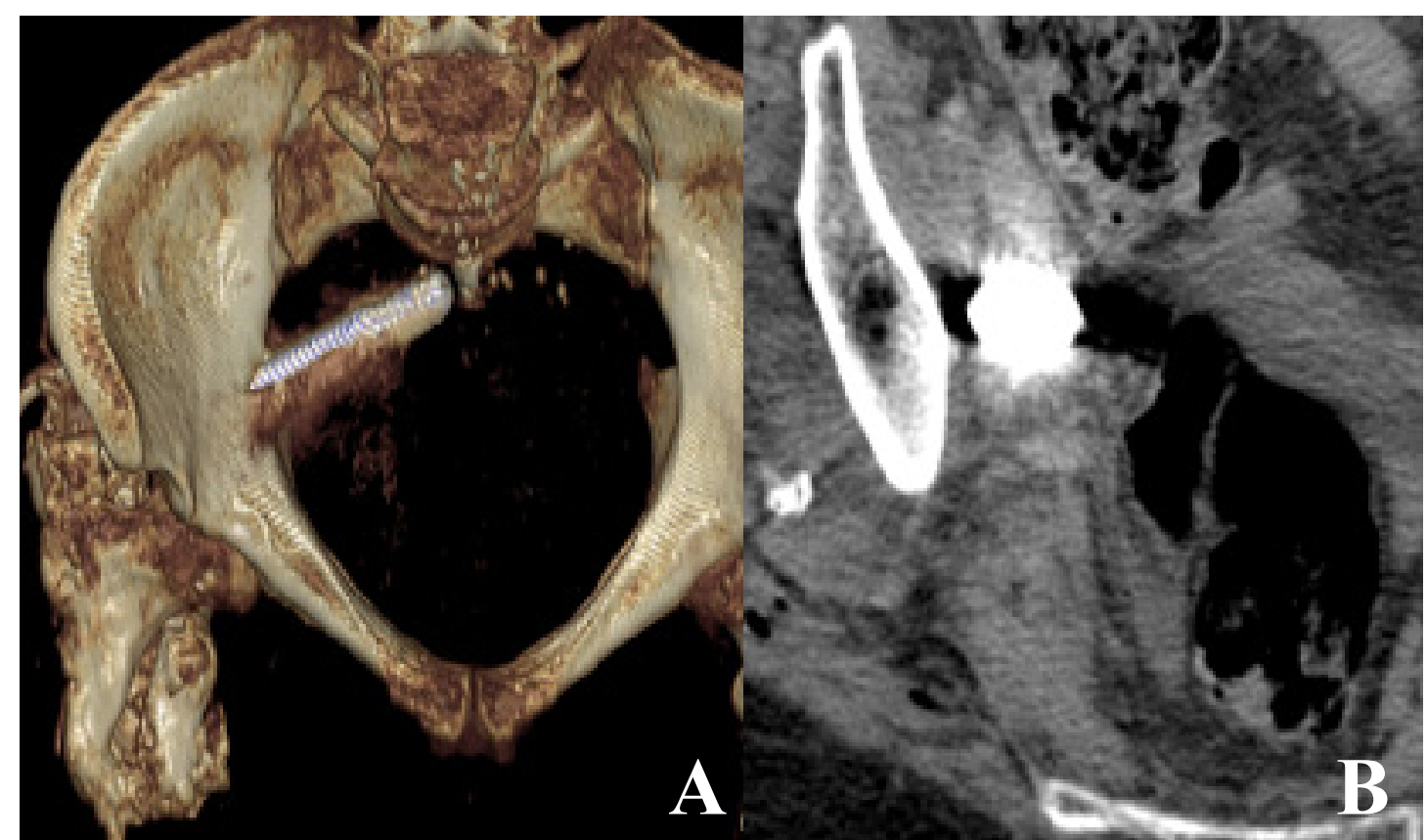

Figura 5. Imágenes tridimensionales de la localización y trayecto. 
en su totalidad intrapélvica.

El rescate quirúrgico del tornillo fue ágil, sin resistencia ósea alguna, localizado en la vecindad de la cara anterior del asa intestinal rectal, sin lesionar tejidos ni complicaciones adicionales (figura 6).

En la figura 7 se muestra erosiones en el tornillo prisionero cérvico-cefálico extraído.

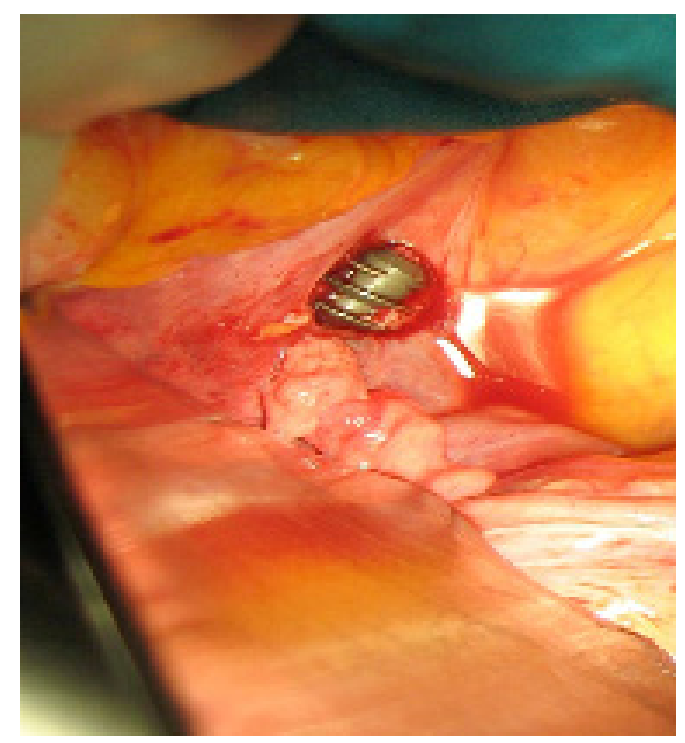

En la revisión a los 6 meses de la segunda cirugía, la radiología de control muestra (figura 8) una integridad cérvico-cefálica que acompaña a la amplia consolidación del foco, aún con cierta osteoporosis proximal y calcificaciones heterotópicas. La marcha es normal con alza, muleta de apoyo, cierta cojera aunque indolente y satisfecha. Completó el plan rehabilitador iniciado en el primer proceso quirúrgico.

Figura 6 (izquierda). Evidencia del clavo en la laparotomía abdominal. Figura 7 (derecha). Erosiones marginales del tornillo CC.

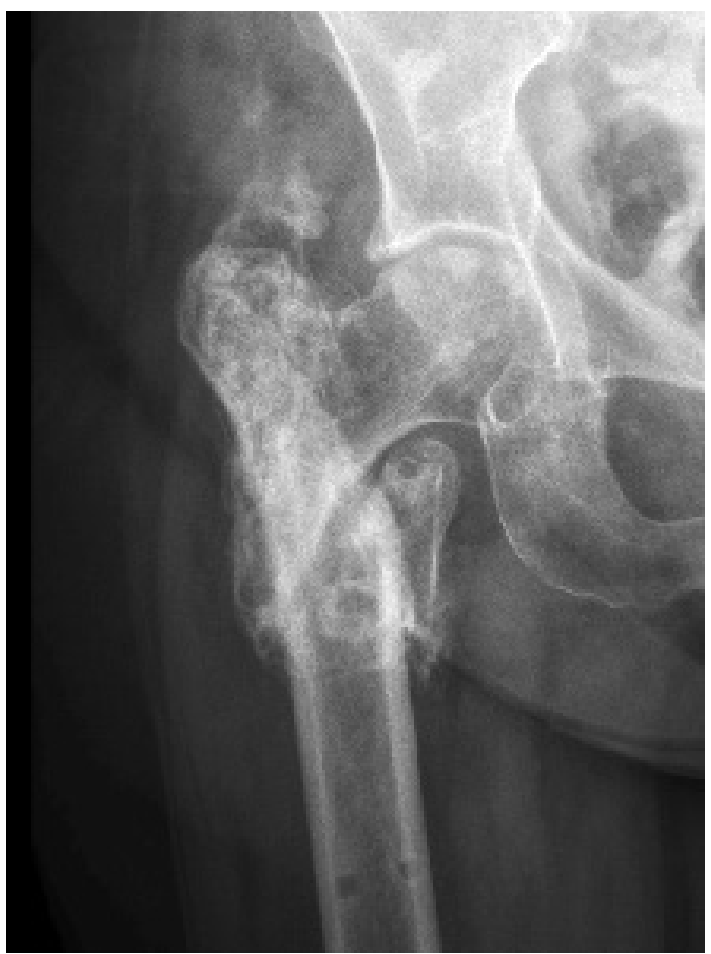

Figura 8. Control radiológico a los 6 meses. 


\section{Discusión}

No nos sorprenden los factores adversos que pensamos han concurrido en este caso -la mala calidad ósea o escasa resistencia del hueso, patrón o trazo de la fractura, edad de la paciente, la calidad de la reducción, diseño del implante y, las limitaciones técnicas aprendidas del error tales como la inserción centro-centro en el cuello y cabeza femorales, excesiva vecindad al subcondral cefálico - por debajo de $25 \mathrm{~mm}$, según preconiza Baumgärtner [5] citado por Carpintero [3]-, que nos elevan las posibilidades de obtener complicaciones postoperatorias tardías.

La reflexión la orientamos por un lado en el insuficiente bloqueo del tornillo prisionero que -a tenor de las evidencias de las erosiones metálicas halladas en el margen del acanalado superior del tornillo protagonista (figura 7)-, la falta de esos 4- $6^{\circ}$ de rotación que hubieran ofrecido una libre entrada en esta ranura superior ad hoc; de otro, la carga parcial prematura que nos enseña a dar cumplido y paciente margen a los inicios de la marcha.

El colapso del foco fracturario se suele producir de forma progresiva en las primeras semanas, consiguiendo en el peor de los casos la varización secundaria grave de la cadera -entre 3,5\%[8] y $8,3 \%$ [9] según autores-, la salida del clavo (habitual hacia lateral)[7], el no menos frecuente cutout que oscila entre el 2-4\% -del 1,7\% de Aune[9] al $4,6 \%$ de Marqués[10]- $y$, de otros que merecen citarse tales como el 2,9\% [8], o el 4\% [11,12].

La excepcional -por infrecuente- migración centrípeta intrapélvica (cut-in), tal es nuestro caso y el de otros autores $[2,6,13]$ resulta ser un proceso adverso -por fortuna- contemplado en la literatura con excepcional rareza.

El cut-out es por lo general un proceso multifactorial o complicación tardía que nos fuerza al rescate y con mayor rigor en el caso del cut-in. La colocación del tornillo "protagonista" en la vecindad del hueso subcondral, o en su defecto en una posición inferior o posterior del cuello, es un gesto principal para prevenir el cut-out tardío.

Sin duda, este proceso nos viene a ilustrar un final de la mejor de las maneras, con una gran tolerancia del enfermo a nuestra explicación de las desafortunadas complicaciones, habiendo evitado hasta el momento actual una artroplastia precisa, aunque aún no necesaria.

\section{Referencias}

1. Orozco R, Sales JM, Videla M. Atlas de osteosintesis. Barcelona. Ed. Masson. 1998.

2. Malkani A, Karandikar N. Revision fixation for failed intertrochanteric fractures. Tech. Orthop. 2002; 17(4):443-447.

3. Carpintero BP. ¿Qué hay de nuevo en el tratamiento de las fracturas trocantéreas?. Med \& Mark Comm. 2009;3-13.

4. Adams CL, Robinson CM, Court-Brown CM, McQueen MM. Prospective randomized controlled trial of an intramedullary, versus dynamic hip screw and plate for intertrochanteric fractures of the femur. J. Orthop. Traum 2001;15:394-400.

5. Baumgärtner MR, Curtis SL, Lindskorg DM, Keggl JM. The value of the tip-apex distance in predicting failure of fixation of pertrochanteric fractutres of the hip. J. Bone Joint Surg. Am. 1995;77:1058-1064.

6. Merino RML, Fernández MJM, Urbano LV. Cut-in de clavo gamma. Arch. Clin-Quirur. Traum. Cadera. 2009;1:38-43.

7. Kellam JF. Tratamiento quirúrgico de las fracturas pertrocantéreas. En: Sledge CB. Cadera. Ed Marban 1999;6:109-137.

8. Sanjuan-Cerveró R, Morales S-VM, SanchisSanegre JR, Fenollosa-Gómez J. Comparación clínica entre clavo Gamma y clavo Claufitt en fracturas inestables de fémur proximal. Rev.Ortop Traumatol 2007;51:325-334.

9. Aune AK, Ekeland A, Odegaard B, Grogaard B, Alho A. Gamma nails vs. compresion screw for trochanteric femoral fractures. 15 reoperations in a prospective, randomized study of 378 patients. Acta Orthop Scand 1994;65:127-130.

10. Marques F, Pelfort X, Garcías-Casas O, Ramírez M, Aeon A, Ballester J. Estudio prospectivo aleatorio comparativo del tornillo deslizante y el clavo Gamma en el tratamiento de las fracturas pertrocantéreas. Rev Ortop Traumatol 2002;46:505-509.

11. Valverde JA, Alonso MG, Porro JG, Rueda D, Larrauri PM, Soler JJ. Use of the Gamma nail in the treatment of fractures of the proximal femur. Clin Orthop Rel Res 1998;350:56-61.

12. Bertrand D, González-Busto I, Solís A, PazAparicio J, Núñez-Batalla D, Paz-Jiménez J. Fracturas pertrocantéreas de fémur en el anciano: clavo Gamma y clavo PFN. Estudio comparativo. Rev Ortop Traumatol 2004; 48:22-30.

13. Lorich D, Geller D, Nielson J. Osteoporotic pertrochanteric hip fractures. J. Bone Joint Surg. 2004;86 Suppl A:398-410.

14. Gomar SF. Las fracturas de cadera en el anciano desde el punto de vista sociosanitario. Discurso de recepción del académico electo. Academia de Medicina Comunidad Valenciana. 25 de mayo 2004. 